Original Article

\title{
IN VITRO IN VIVO EVALUATION OF NIOSOMAL FORMULATION OF FAMOTIDINE
}

\section{RIZWANA KHAN ${ }^{*}$, RAGHUVEER IRCHHAIYA ${ }^{2}$}

${ }^{1}$ Department of Pharmaceutics, Monad University, Hapur, U. P. India, ${ }^{2}$ Institute of Pharmacy, Bundelkhand University, Jhansi, 284128, U. P. India

Email: rizwana9506@gmail.com

Received: 30 Oct 2019, Revised and Accepted: 06 Jan 2020

\section{ABSTRACT}

Objective: The present study was aimed on formulation and evaluation of famotidine loaded niosomal formulation for in vitro and in vivo pharmacokinetic behaviour. Formulating it as niosomal formulation might be quite advantageous for prolonging the duration of pharmacological action and improved bioavailability.

Methods: In the present study niosomal formulations were prepared by using most documented thin film hydration technique by using various grades of surfactants (span 20,40,60,80) in varying ratios with cholesterol, negative charge inducer di cetyl phosphate (DCP) and drug famotidine. Suitable preformulation studies were conducted like identification of drug, excipient and drug compatibility study. The optimized drug loaded niosomes were characterized for size and morphology, polydispersity index, zeta potential, drug entrapment, in vitro release, in vivo study and stability study.

Results: The results showed that the vesicles formed were spherical in shape, size ranging between $160.1 \mathrm{~nm}$ to $718.7 \mathrm{~nm}$ with zeta potential values indicating good stability and formulation containing span 60 (NMS7) showed the highest entrapment efficiency (73.234\%). All the formulations showed prolonged release profile for more than $24 \mathrm{~h}$ with release kinetics better suited to zero order release pattern. In vivo study conducted on rabbits predicted a fourfold increase in pharmacokinetic parameter (area under curve)AUC and pharmacological action for more than $24 \mathrm{~h}$ as compared to free drug famotidine which showed its action only upto $12 \mathrm{~h}$.

Conclusion: Thus the famotidine loaded niosomal formulation may be considered as a very promising drug delivery system which could be successfully employed for prolonging the drug release and overcoming the drawbacks of conventional drug delivery systems.

Keywords: Famotidine, Niosomes, Cholesterol, Surfactant

(C) 2020 The Authors. Published by Innovare Academic Sciences Pvt Ltd. This is an open access article under the CC BY license (http://creativecommons.org/licenses/by/4.0/) DOI: http://dx.doi.org/10.22159/ijpps.2020v12i3.36210. Journal homepage: https://innovareacademics.in/journals/index.php/ijpps

\section{INTRODUCTION}

In recent years transporting the drug molecule to desired site in the biological system has become a very sophisticated area of pharmaceutical research, the role of novel drug delivery is not only limited to ease of administration and convenience but along with this it is also needed to provide better pharmacological effect along with reduced side effects. These novel carriers provide sustained drug release for prolonged duration thus resulting in enhanced therapeutic efficacy and minimized side effects [1].

From the last few years, liposomes have attracted a great attention in the delivery of drugs because of the many advantages, e. g. they are biodegradable, non-toxic, amphiphilic in nature, penetration enhancers and effective in the modulation of drug release properties. But liposomes exhibit some difficulties, including the instability of aqueous dispersions on storage and the leakage of the encapsulated drugs, the high cost of synthetic phospholipids and variable purity of natural phospholipids. An alternative approach that overcomes several of these problems associated with liposomes involves formation of liposome-like vesicles (niosomal dispersions) from non-ionic surfactants, commonly referred as niosomes [2].

These niosomal dispersion are formed from self-assembly of hydrated synthetic non-ionic surfactant monomers which are capable of entrapping a wide variety of drugs both hydrophilic and hydrophobic, can be delivered by various routes like oral, transdermal, parenteral, occular [3] and have been considered superior to microspheres, nanoparticles, liposomes and other carriers in terms of better entrapment of drugs, better target site specificity and in handling burst effect of drug release $[4,5]$. Niosomes have also shown advantages as drug carriers, such as being low cost and chemically stable as compared to liposomes [6].

Famotidine is a potent $\mathrm{H} 2$ receptor antagonist. It is widely prescribed in gastric ulcers, duodenal ulcers, zollinger Ellison syndrome and gastro oesophageal reflux disease. Compared with other drugs of this class it is 7.5 to 20 times more potent than ranitidine and cimetidine respectively [7]. Inspite of its benefits it suffers from low and variable bioavailability which is $20-40 \%$ and short biological half-life $(2.5-4 \mathrm{~h})$. Conventional formulations of 20 mg dose can inhibit gastric acid secretion hardly upto 5 to $7 \mathrm{~h}$. Thus formulating it into niosomal formulation is expected to provide a prolonged pharmacological effect with reduced dosing frequency and better patient compliance by reducing the peak and valley curves in the plasma drug concentration profile. It is also expected to provide some local effect on the stomach and small intestine [8]. Thus the aim of the present research was to prepare drug loaded niosomes of famotidine for oral controlled release by using different grades of span like Span 20,40,60,80 by thin film hydration technique and also optimizing the different process variables and further its characterization by various In vitro and In vivo methods.

\section{MATERIALS AND METHODS}

Famotidine was obtained as gift sample from Glenmark Pharmaceutical Ltd (India), various other chemicals like cholesterol was purchased from Loba Chemie Pvt. Ltd. (India), chloroform, methnol, Span 20,40,60,80 were obtained from Central Drug House, Delhi, (India), di cetyl phosphate (DCP) was purchased from Sigma Aldrich, and dialysis membrane from Himedia (India). All chemicals used in the study were of analytical grade.

\section{Identification of drug}

\section{Ultraviolet spectroscopy}

UV Spectral Analysis was carried out for the identification of famotidine. Different concentrations of Famotidine in the range 2 to $20 \mu \mathrm{g} / \mathrm{ml}$ were scanned for $\lambda_{\max }$ between $200-400 \mathrm{~nm}$ using Double Beam Spectrophotometer (Shimadzu UV-1700 series). 


\section{Fourier transforms infrared spectroscopy (FTIR)}

FTIR spectra of famotidine, from $4000 \mathrm{~cm}^{-1}$ to $450 \mathrm{~cm}^{-1}$, was obtained using FTIR spectrophotometer (Perkin Elmer) according to $\mathrm{KBr}$ pellet method and compared with standard reference spectra of famotidine. The drug excipients interaction study was also carried out by IR spectroscopy. The IR spectrum of combination of drug and various excipients to be used in the formulation was obtained using FTIR spectrophotometer and compared with the individual spectra of drug and excipients to investigate any interactions. Spectral analysis using IR helped to identify any modification in the characters of the formulation when it was mixed with the non-drug substances [9].

\section{Solubility}

To obtain a desirable concentration of the drug in blood, solubility plays a major role (Baby et al. 2012) Solubility studies of famotidine were carried out in distilled water, $0.1 \mathrm{~N} \mathrm{HCl}(\mathrm{pH} 1.2)$ and phosphate buffer (pH 7.4). Saturated solutions were prepared in screw capped tubes by adding excess drug to the solvents and shaking on the shaker (Jyoti Scientific Industry, Gwalior) for $24 \mathrm{~h}$ at $25 \pm 0.5^{\circ} \mathrm{C}$. The solutions were then filtered, diluted and analyzed by UV Spectrophotometer (Shimadzu UV-1700 series, Japan). The determinations were made in triplicate shown in (table 1).

\section{Method of preparation}

In the present study, niosomal formulations were prepared by thin film hydration technique as reported earlier with slight modifications [10] by using different grades of span (span 20, span 40, span 60, and span 80 ) at various cholesterol: surfactant ratios, given in (table 2) (Where NML is (batch A) containing span 20, NMP is (batch B) containing span 40, NMS is (batch C) containing span 60 and NMO is (batch D) containing Span 80). DCP was added to the formulation that acts as a negative charge inducer which provides more efficient drug delivery and keeps the niosomal formulation stable for long period of time. Accurately weighted quantities of surfactants, cholesterol and drug were taken and were dissolved in chloroform-methanol mixture in a round bottom flask and DCP was added to the above mixture. The chloroform methanol mixture was evaporated in a rotary flask evaporator under a vacuum at temperature of $60^{\circ} \mathrm{C}$ at $120 \mathrm{rpm}$ until a smooth, dry lipid film was obtained.

Further preparation flask was kept in vacuum desiccators overnight for complete removal of chloroform and mehanol as discussed by [11]. Then film was hydrated with aqueous phase (PBS pH 7.4) for 3 $\mathrm{hr}$ at room temperature with shaking. The niosomal suspension was kept at $2-8{ }^{\circ} \mathrm{C}$ for $24 \mathrm{hr}$. These preparations were optimized on the basis of entrapment efficiency.

Table 2: Composition of niosomal formulations with different grades of span at varying concentration of surfactant and cholesterol

\begin{tabular}{|c|c|c|c|c|c|}
\hline Formulation code & Surfactant (Mg) & Cholesterol (mg) & DCP (Mg) & Chloroform/methanol & Drug (mg) \\
\hline $\mathrm{NML}^{\mathrm{a}} 1 / \mathrm{NMP}^{\mathrm{b}} 1 / \mathrm{NMS}^{\mathrm{c}} 1 / \mathrm{NMO}^{\mathrm{d}} 1$ & 100 & - & - & $1: 4$ & 5 \\
\hline NML2/NMP2/NMS2/NMO2 & 90 & 10 & - & $1: 4$ & 5 \\
\hline NML3/NMP3/NMS3/NMO3 & 80 & 20 & - & $1: 4$ & 5 \\
\hline NML4/NMP4/NMS4/NMO4 & 70 & 30 & - & $1: 4$ & 5 \\
\hline NML5/NMP5/NMS5/NMO5 & 60 & 40 & - & $1: 4$ & 5 \\
\hline NML6/NMP6/NMS6/NM06 & 50 & 50 & - & $1: 4$ & 5 \\
\hline NML7/NMP7/NMS7/NMO7 & 47.5 & 47.5 & 5 & $1: 4$ & 5 \\
\hline NML8/NMP8/NMS8/NMO8 & 60 & 30 & 10 & $1: 4$ & 5 \\
\hline NML9/NMP9/NMS9/NMO9 & 50 & 30 & 20 & $1: 4$ & 5 \\
\hline
\end{tabular}

${ }^{a} \mathrm{NML}$ is batch containing span $20,{ }^{\mathrm{b}} \mathrm{NMP}$ is batch containing Span $40,{ }^{\mathrm{c}} \mathrm{NMS}$ is batch containing span 60 and ${ }^{\mathrm{d}} \mathrm{NMO}$ is batch containing span 80

\section{Particle shape and morphology by transmission electron microscopy (TEM)}

The shape and morphology of prepared optimized niosomes were evaluated by optical microscopy and by Transmission electron microscopy (Jeol JEM1400 Tokyo Japan) at Central Drug Research Institute (CDRI Lucknow).

\section{Scanning electron microscopy (SEM)}

The vesicle formation were examined by scanning electron microscopy (Carl ZEISS microscopy Ltd) at central instrument facility at (Banaras Hindu university) IIT-BHU), in order to determine size, shape and lamellarity.

\section{Entrapment efficiency}

An aliquot of the freshly prepared purified niosomal dispersion $(5$ $\mathrm{mg} / \mathrm{ml}$ ) was diluted with $10 \%$ Triton X-100 in a ratio of $1: 99 \mathrm{vol} / \mathrm{vol}$ for $5 \mathrm{~min}$. The detergent dissolved the niosomes and yielded a clear solution. The resultant was centrifuged to get a clear supernatant. The supernatant was suitably diluted with phosphate buffer and analysed for Famotidine concentration using UV spectrophotometer at $\lambda$ max.266 nm to calculate the amount of entrapped drug. The Percentage of entrapped Famotidine was calculated by applying the following equation $[12,13]$.

$$
\text { Entrapment efficiency } \%=\frac{\text { amount of entrapped drug }}{\text { Initial amount of drug added }} \times 100
$$

\section{Vesicle size, zeta potential and polydispersity index determination}

Niosomal suspension was characterized for vesicle size and vesicle size distribution of the multilamellar vesicles by using Beckman coulter delsa ${ }^{\mathrm{TM}}$ nano zeta potential and submicron particle size analyser at central instrument facility of (IIT-BHU, India). Zeta potential, Size, size distribution measurements and polydispersity index measurements were obtained automatically as the instrument is microprocessor controlled. The instrument uses Photon correlation spectroscopy(PCS) to determine the particle size where as it uses electrophoretic light scattering (ELS) to determine electrophoretic movement of charged particles under an applied electric field from Doppler shift of scattered light, for zeta potential determination.

\section{In vitro drug release study}

The In vitro release of famotidine from the prepared niosomes was carried by membrane diffusion method $[14,15]$. The drug release study was carried out using dialysis membrane. Measured amount of niosomes were placed in the cylinder. This cylinder was fitted at its lower end with dialysis membrane which served as the donor compartment. The glass cylinder was attached to the shaft of the dissolution apparatus and then suspended in the dissolution flask of USP dissolution apparatus paddle type containing $500 \mathrm{ml}$ of phosphate buffer ( $\mathrm{pH}$ 7.4) maintained at $37{ }^{\circ} \mathrm{C}$ which served as the receptor compartment and aliquots were withdrawn at intervals of $2 \mathrm{~h}$ for $24 \mathrm{~h}$. At each sampling time, the volume of receptor compartment was maintained with equal volume of phosphate buffer, $\mathrm{pH} 7.4$ or $0.1 \mathrm{~N}$ HCL to maintain the sink condition during the whole study. The drug in withdrawn samples was estimated by UV spectrophotometer (Shimadzu UV-1700 series, Japan) at $\lambda$ max 266 $\mathrm{nm}$. All assessments were completed in triplicate [16].

\section{Release kinetics}

For the statistical analysis, four principally practised mathematical models were chosen for the estimation of release kinetics of drug from famotidine loaded niosomes ie. Zero order (Eq.1), First order 
(Eq.2), HiguChi square root model (Eq.3) and Kosmeyer Peppas model (Eq.4)

$$
\begin{gathered}
\mathrm{C}=\mathrm{C}_{0}-\mathrm{K}_{0} \mathrm{t} \ldots \ldots .(1) \\
\log \mathrm{C}=\log \mathrm{C}_{0}-\mathrm{k}_{\mathrm{t}} / 2.303 \ldots \ldots(2) \\
\mathrm{Q}=\mathrm{k}_{\mathrm{H}} \mathrm{t}^{1 / 2} \ldots \ldots \text { (3) } \\
\mathrm{M}_{\mathrm{t}} / \mathrm{M}_{\infty}=\mathrm{kt}^{\mathrm{n}} \ldots \ldots \text { (4) }
\end{gathered}
$$

Where $\mathrm{C}$ and $\mathrm{Q}$ is the amount of drug released at time $\mathrm{t}, \mathrm{C}_{0}$ is the initial concentration of drug, $M_{t} / M_{\infty}$ is the fraction of drug released at time $t$ and $\mathrm{k}_{0}, \mathrm{k}, \mathrm{k}_{\mathrm{H}}$ are the corresponding rate constants,, $\mathrm{n}$ is the diffusional release exponent that could be used to characterize the different release mechanism $(n \leq 0.45)$ fckian diffusion, $(0.45<n<1)$ anomalous transport and $(n=1)$ case II transport, zero order release [17-19].

Data obtained from drug release assessment were treated complimenting zero order (cumulative amount of drug release vs time), first order (log cumulative percentage of drug remaining vs time), Higuchi (cumulative percentage of release vs square root of time) and Kosmeyer-Peppas (log cumulative percentage of drug released vs log time) equation models. Excel 2007 was used to evaluate the results of in vitro drug release to acquire the best fit kinetic model for drug release from prepared niosomal formulation [20].

\section{In vivo study}

Nine male white Newzealand rabbits weighing an average weight of $2.5 \mathrm{~kg}$ were selected for the study. The study protocol was approaved by the Institutional Animal Ethical Committee registration no: BU/Pharm/IAEC/a/16/14 for the use of animals in research. The rabbits were fasted overnight or for twelve hours with free access to water. On study days, rabbits were placed in metal restrainers at $9.00 \mathrm{am}$. Doses were administered orally by gavage at $9.00 \mathrm{am}$ as $4 \mathrm{ml}$ bolus of the respective formulations [21]. The studied formulations were free famotidine solutions in normal saline and the freshly prepared niosomal formulations $(4 \mathrm{ml})$ containing both free and entrapped drug. Blood samples were collected at $1,2,4,6,8,10,12$, and $24 \mathrm{~h}$ after administration of free famotidine solution and at $1,2,4,6,8,10,12$ and $24 \mathrm{~h}$ after administration of famotidine niosomal dispersion. The experiment were carried out on same rabbits in which atleast two weeks passed between each application inorder to obtain complete washout of drug.

Blood sample, $2 \mathrm{ml}$ each were collected from the marginal ear vein of the rabbits, into heparinised centrifuge tubes just before dosing and at $1,2,4,6,8,10,12,24 \mathrm{~h}$ during the study. Blood samples were centrifuged at $1500 \mathrm{rpm}$ and the plasma was separated. One undosed plasma sample was kept as a blank. To one $\mathrm{ml}$ of each of the other plasma samples, $5 \mathrm{ml}$ of acetonitrile (to precipitate blood proteins) was added, the tubes were then centrifuged at $2500 \mathrm{rpm}$ for $15 \mathrm{~min}$, $4 \mathrm{ml}$ of the supernatant was pippetted out, to which $0.2 \mathrm{ml}$ of $1.47 \mathrm{M}$ perchloric acid was added and the drug concentration was determined by UV Spectroscopy at $258 \mathrm{~nm}$. The blank consist of $1 \mathrm{ml}$ undosed plasma, $4 \mathrm{ml}$ acetonitrile and $0.2 \mathrm{ml}$ of $1.47 \mathrm{M}$ perchloric acid (to precipitate plasma proteins) [22].
The calibration curve of famotidine was prepared as follows: Famotidine solutions in acetonitrile were prepared at concentration of $1-10 \mu \mathrm{g} / \mathrm{ml}$. One millilitre of this solution was made up to $5 \mathrm{ml}$ with acetonitrile. To each of this solution $1 \mathrm{ml}$ of plasma from undosed rabbit blood was added and contents were centrifuged at $2500 \mathrm{rpm}$ for $15 \mathrm{~min}$. Supernatant $(4 \mathrm{ml})$ was then pippetted out to which $0.2 \mathrm{ml}$ of $1.47 \mathrm{M}$ perchloric acid was added and the absorbance was measured at $\lambda_{\max } 258 \mathrm{~nm}$. The blank was prepared using plasma from the undosed animal, acetonitrile and perchloric acid in exactly the same way as the calibration curve for famotidine was plotted as absorbance at $\lambda_{\max } 258 \mathrm{~nm}$ versus concentration was linear over the range of $1-10 \mu / \mathrm{ml}$ with the correlation coefficient of 0.999 [23].

\section{Stability studies}

\section{Drug leakage studies}

The multilamellar vesicles prepared by thin film hydration were subjected to stability studies. After measuring the initial percentage entrapment of the drug in formulations, four batches of the same formulation were stored in sealed glass ampoules ( 1 each) at $4 \pm 2^{\circ} \mathrm{C}$ $25 \pm 2^{\circ} \mathrm{C} 37 \pm 2^{\circ} \mathrm{C}$ for a period of atleast one month. After every seven days, percentage entrapment of drug was determined in the formulation to know the amount of drug leaked out. The percentage drug lost was calculated taking the initial entrapment of drug as $100 \%$. Plain niosomal suspension (without drug) prepared and stored in the same way, served as blank for this purpose, also after each week the formulation was observed microscopically and visually to check for the number of disrupted vesicles (in a constant area of slide visualized through microscope) and for their sedimentation and aggregation behaviour.

\section{RESULTS AND DISCUSSION}

\section{Identification of drug by ultraviolet spectroscopy}

UV spectrum of famotidine was obtained by scanning Different concentrations of Famotidine in the range 2 to $20 \mu \mathrm{g} / \mathrm{ml}$ between 200-400 nm using Double Beam Spectrophotometer (Shimadzu UV1700 series). The $\lambda_{\max }$ was found to be $266 \mathrm{~nm}$.

\section{Identification of drug by IR spectroscopy}

The IR spectrum of pure drug famotidine showed characteristic peaks at $(3506.10,3401.17)$ for $\mathrm{N}-\mathrm{H}$ stretching, (1329.91Asy, 1145.70 Sym) stretching vibration for sulphones, (2936.98) C-H stretching, (1643.57) N-H Bending, (1534.10) N - H Bending $\left(\mathrm{NH}_{2}-\right.$ $\mathrm{SO}_{2}$ ) which matched with the reference spectra thus confirming the purity of the compound. The drug excipient interaction study showed no significant interaction with any of the excipients used in the formulation.

\section{Solubility determination}

Preliminary solubility study of drug famotidine was conducted in various solvents like distilled water, $0.1 \mathrm{~N} \mathrm{HCl}$ and phosphate buffer PBS pH 7.4 which showed that its solubility in $0.1 \mathrm{~N} \mathrm{HCl}$ was appreciable. The results are shown in table 1 .

Table 1: Solubility of famotidine in various solvents

\begin{tabular}{lll}
\hline S. No. & Solvents & *Solubility $(\mathbf{m g} / \mathbf{m l}))$ \\
\hline 1. & Distilled water & $0.367 \pm 0.071$ \\
2. & PBS pH 7.4 & $0.566 \pm 0.211$ \\
3. & $0.1 \mathrm{~N} \mathrm{HCl}$ & $7.442 \pm 0.312$ \\
\hline
\end{tabular}

$\left({ }^{*}\right.$ All values are expressed as mean $n=3, \pm$ SD: standard deviation)

\section{Particle shape and morphology by TEM}

The preliminary information regarding the shape and morphology of niosomal formulation was determined by optical microscopy which revealed the spherical shape of niosomes but unfortunately the information concerning microstructure of noisome could not be visualized due to low magnification power of microscope. Therefore TEM was employed to elucidate morphology of the niosomal vesicles. TEM photo micrographs confirm that the niosomes have a closed spherical shape shown in fig. 1 . The size range of the prepared niosomes was in nano range.

\section{Scanning electron microscopy}

The SEM photomicrographs of famotidine loaded niosomes (fig. 2) revealed spherical shape of niosomes with no aggregation of vesicles and the size range was found to be in nanometer which is well suited for oral delivery of famotidine loaded niosomes. 


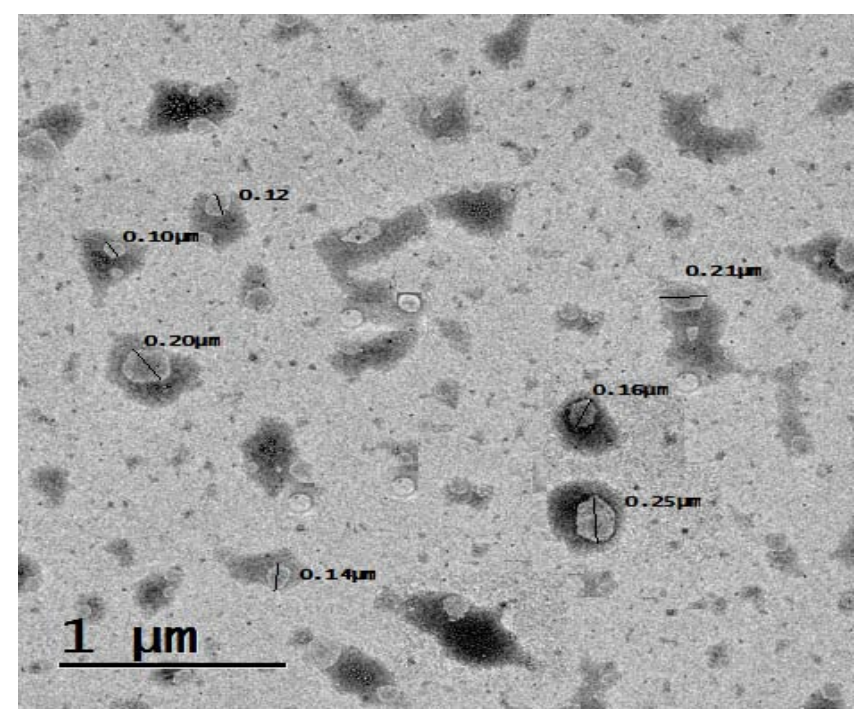

Fig. 1: TEM photomicrographs of famotidine loaded niosomal formulation

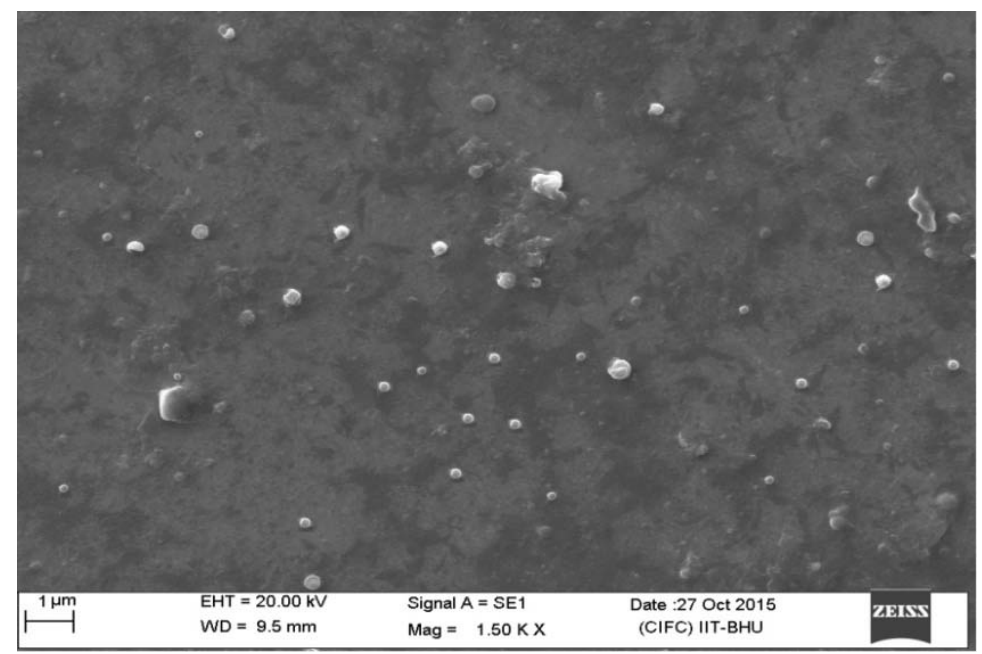

Fig. 2: SEM images of famotidine loaded niosomal formulation

\section{Entrapment efficiency}

On the basis of entrapment efficiency values for all the prepared batches of famotidine loaded niosomes, formulations NML7, NMP7, NMS7 and NM07 were selected as optimized formulations as they showed better \% entrapment as compared to other batches. Famotidine encapsulation efficiency of optimized formulations are given in (table 3) which clearly shows that the formulation containing span 60 showed the highest entrapment efficiency of $73.234 \%$ followed by span 40 and span 20 containing formulation ie. $71.308 \%$ and $69.333 \%$ respectively. While span 80 containing formulation showed the least entrapment efficiency ie. $65.086 \%$ these results were in accordance with previous study done by Hao and $\mathrm{Li}$ (2011) [25], this could be attributed to the structure, orientation and packing behaviour of the surfactant. Span 20, 40 and 60 have the same head group but different alkyl chain but span 80 has an unsaturated alkyl chain. The introduction of double bonds made the chain bend. This means that the adjacent molecules cannot be tight when they form membrane of niosomes. This caused the membrane to be more permeable which possibly explains the low entrapment efficiency behaviour. While span 60 has the longest saturated alkyl chain due which it showed highest entrapment efficiency. The increase in the alkyl chain length of different grades of span led to increase in the encapsulation efficiency (\%EE) [24, 25]. The higher entrapment in case of span 60 can also be due to the solid nature, hydrophobicity and high phase transition temperature of the span60 [26]. Spans 60 with the highest transition temperature $\left(\mathrm{Tc}=53^{\circ} \mathrm{C}\right)$ amongst all spans $\left(16^{\circ} \mathrm{C}\right.$ for span $20,42^{\circ} \mathrm{C}$ for span 40 and $-12{ }^{\circ} \mathrm{C}$ for span 80) [27]. Exhibit highest entrapment efficiency [28]. Gel transition temperature of span increases as the length of acyl chain increases. For example span 20 with 9 carbon atoms in the carbon chain is liquid at room temperature [29]. Interestingly with increasing carbon chain length entrapment efficiency increases [30]. Moreover HLB of the surfactant also affects the entrapment efficiency, as it was observed that lower the HLB of the surfactant higher was the entrapment efficiency and stability. In the present research niosomes prepared using span 60 (HLB 4.7) exhibited higher entrapment as compared to span 20 with higher HLB value (HLB 8.6) [31, 32].

\section{Vesicle size, zeta potential and polydispersity index}

On the basis of above findings the formulation NML7, NMP7, NMS7 and NM07 were selected for further studies. The vesicle size of various developed niosomal formulations with various grades of span were determined by submicron particle size analyser. It was clearly observed from the results that the mean vesicle size of niosomes containing span 20 were found to be higher as compared to other niosomal formulations containing span 40,60 and 80. The vesicle size of niosomal formulation were obtained in the range of 160.1 $\pm 69.7 \mathrm{~nm}$ to $718.7 \pm 749.9 \mathrm{~nm}$ (given in table 3 ) The vesicle size of niosomes decreased consistently from span 20 to span 80 and are 
found in the following order-span 20>span 40>span60>span 80 which was in conformance with Asthana et al. (2016) [5].

This might be due to increase in hydrophobicity of the surfactant from span 20 to span 80 . The decrease in surface free energy with increasing hydrophobicity of surfactant may be the major reason for reduction in the particle size of niosomes [33]. The zeta potential values (table 3) indicate that all the optimized formulations have good stability, it was also observed that highest zeta potential values were obtained in case of span 20 formulation whereas lowest zeta potential for span 80 formulation which could also be due to increase in hydrophobicity of surfactant (Balakrishnana et al. 2009) [10].

Polydispersity index PDI is used to describe the degree of nonuniformity of a size distribution of particles. Also known as the hererogeneity index. This index is dimensionless and scaled such that values smaller than 0.05 are mainly seen with highly monodisperse standard. PDI value bigger than 0.7 indicate that the sample has a very broad particle size distribution. The numerical value of PDI ranges from 0.0 for perfectly uniform sample with respect to particle size whereas a value approaching 1.0 represents a highly polydisperse sample with multiple particle size populations. In drug delivery applications using lipid based carriers such as liposomes and niosomes formulations, a PDI of 0.3 and below is considered to be acceptable and indicates a homogenous population of phospholipid vesicles [34-36] The optimized niosomal formulations showed polydispersity index in the range of 0.114 to 0.514 given in (table 3 ) indicating the homogeneity of the formulations.

Table 3: Percentage entrapment efficiency, vesicle size, polydispersity index and zeta potential values of optimized niosomal formulations of famotidine

\begin{tabular}{llll}
\hline Formulation code & *oEntrapment & *vesicle size $(\mathbf{n m})$ & Polydispersity index \\
\hline NML7 & $69.333 \pm 0.925$ & $718.7 \pm 749.9$ & 0.514 \\
NMP7 & $71.3085 \pm 0.688$ & $487.6 \pm 52.1$ & 0.114 \\
NMS7 & $73.234 \pm 0.365$ & $236.1 \pm 65.3$ & 0.274 \\
NMO7 & $65.086 \pm 0.938$ & $160.1 \pm 69.7$ & 0.295 \\
\hline
\end{tabular}

(*All values are expressed as mean $n=3, \pm S D$ : standard deviation)

\section{Drug release profile of optimized famotidine loaded niosomal formulation}

The in vitro release study (fig. 3) are often performed to predict how a delivery system might work in ideal conditions and this might also give some indication of its in vivo performance. In present research famotidine encapsulated niosomes were analysed for its in vitro release in $0.1 \mathrm{~N} \mathrm{HCl}$ and in phosphate buffer $\mathrm{pH} 7.4$ which showed constant drug release with no burst effect indicating that the drug was homogeneously dispersed and there was no significant amount of drug adsorbed onto the surface of niosomal vesicles. Whereas the free drug elicited a fast drug release ie. $74.625 \%$ just after $1 \mathrm{~h}$ as compared to niosomal formulations which showed drug release upto $5 \%$ after $1 \mathrm{~h}$. The free drug solution began to plateau only after $4 \mathrm{~h}$ but the optimized niosomal formulations continued to show drug release for more than $24 \mathrm{~h}$ without reaching plateau. This is indicative of rate controlling nature of niosomes as lipid bilayers serve as barrier for drug release.

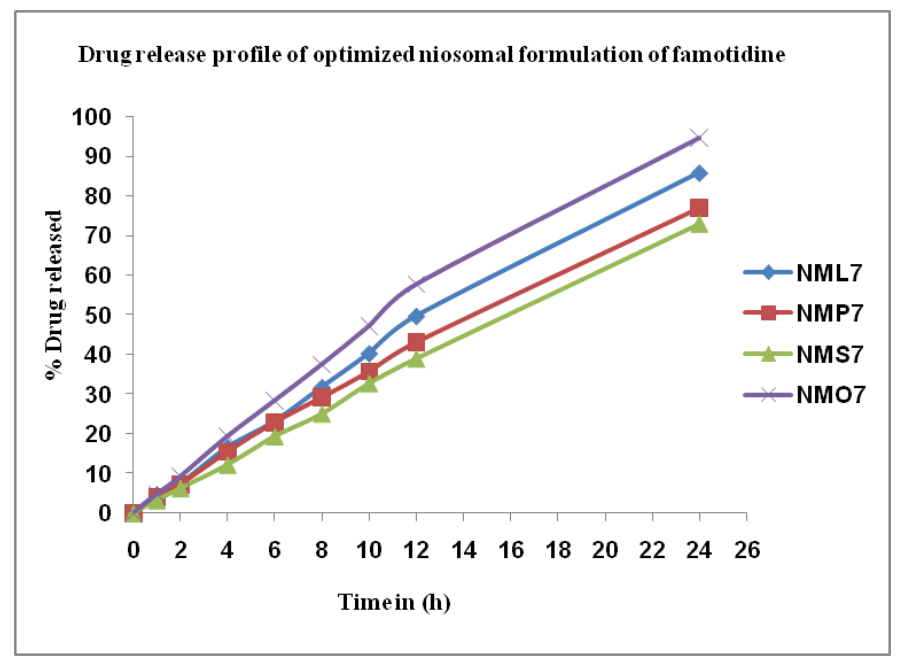

Fig. 3: Drug release profile of optimized niosomal formulation of famotidine (all values are expressed as mean $n=3$ )

\section{Data treatment}

Inorder to study the mechanism of drug release from the niosomal formulation the in vitro release study data were fitted to various mathematical models. The kinetic models used were zero order, first order, Higuchi's square root of time and Kosmeyer and Peppas model (table 4). Famotidine release from niosomal formulation showed a better fit to zero order release pattern furthermore the release pattern when fitted to Kosmeyer Peppas model showed release exponent $(\mathrm{n})$ values ranging from 0.567 to 0.598 which were close to Fickian diffusion pattern. Whereas Formulation with Span 20 showed $n$ value ie 0.618 thus it follows anomalous diffusion mechanism.

\section{In vivo study}

On the basis of in vitro characterization results NMS7 formulation was found to be the most satisfactory one and hence it was selected for further in vivo study. The concentration of drug in plasma at different time interval was determined spectrophotometrically (fig. 4). Different pharmacokinetic parameters such as $C_{\max }$ (maximum concentration of the drug in the blood), $\mathrm{T}_{\max }$ (the time it takes for a drug to reach $\mathrm{C}_{\max }$ ) and AUC area under curve were calculated from the data and the results are represented in (table 5). Analysis of variance (ANNOVA) was employed in the statistical analysis of the determined parameters in this study by using the software SYSTAT 12 . The obtained results indicate $\mathrm{a} * \mathrm{P}$ value $=0.03$ which is less than 
0.05 which clearly indicate that the differences are statistically significant with a ${ }^{*} \mathrm{P}$ value $<0.05$. These clearly indicate a fourfold increase in the area under curve of prepared niosomal formulation of famotidine as compared to free drug solution. This indicate that the niosomal formulation provided prolonged and controlled delivery of drug without any sharp peaks and troughs. Thus besides increasing the patient compliance because of once a day administration, the optimized formulation will be able to reduce the incidence of problems reported due to sharp rise and fall in plasma drug concentrations of famotidine.

Table 4: Release rate constant $(K)$ and correlation coefficient $\left(R_{2}\right)$ obtained after treating the release profile according to various mathematical models

\begin{tabular}{|c|c|c|c|c|c|c|c|c|}
\hline \multirow{2}{*}{$\begin{array}{l}\text { Formulation } \\
\text { code }\end{array}$} & \multicolumn{2}{|l|}{ Zero order } & \multicolumn{2}{|l|}{ First Order } & \multicolumn{2}{|c|}{ Higuchi's square root } & \multicolumn{2}{|c|}{ Kosmeyer Peppas } \\
\hline & $*^{*} \mathbf{K}_{\mathbf{0}}$ & $* \mathbf{R}^{2}$ & ${ }^{*} \mathbf{K}_{1}$ & ${ }^{*} \mathbf{R}^{2}$ & $*^{*} \mathbf{K}_{\mathbf{H}}$ & ${ }^{*} \mathbf{R}^{2}$ & ${ }^{*} \mathbf{K}_{\mathrm{k}}$ & $* \mathbf{R}^{2}$ \\
\hline NML7 & $0.0603 \pm 0.0007$ & $0.992 \pm 0.001$ & $0.0011 \pm 0.000$ & $0.641 \pm 0.008$ & $2.3067 \pm 0.0243$ & $0.912 \pm 0.004$ & $0.780 \pm 0.013$ & $0.950 \pm 0.007$ \\
\hline NMP7 & $0.0534 \pm 0.0007$ & $0.994 \pm 0.001$ & $0.0010 \pm 0.000$ & $0.636 \pm 0.007$ & $2.0495 \pm 0.0238$ & $0.919 \pm 0.003$ & $0.795 \pm 0.011$ & $0.955 \pm 0.005$ \\
\hline NMS7 & $0.0510 \pm 0.0004$ & $0.998 \pm 0.001$ & $0.0011 \pm 0.000$ & $0.676 \pm 0.005$ & $1.9306 \pm 0.0145$ & $0.897 \pm 0.004$ & $0.761 \pm 0.020$ & $0.934 \pm 0.012$ \\
\hline NM07 & $0.0663 \pm 0.0003$ & $0.985 \pm 0.000$ & $0.0011 \pm 0.000$ & $0.608 \pm 0.009$ & $2.5704 \pm 0.0171$ & $0.928 \pm 0.004$ & $0.811 \pm 0.013$ & $0.965 \pm 0.005$ \\
\hline
\end{tabular}

$(*$ All values are expressed as mean $n=3, \pm$ SD: standard deviation)

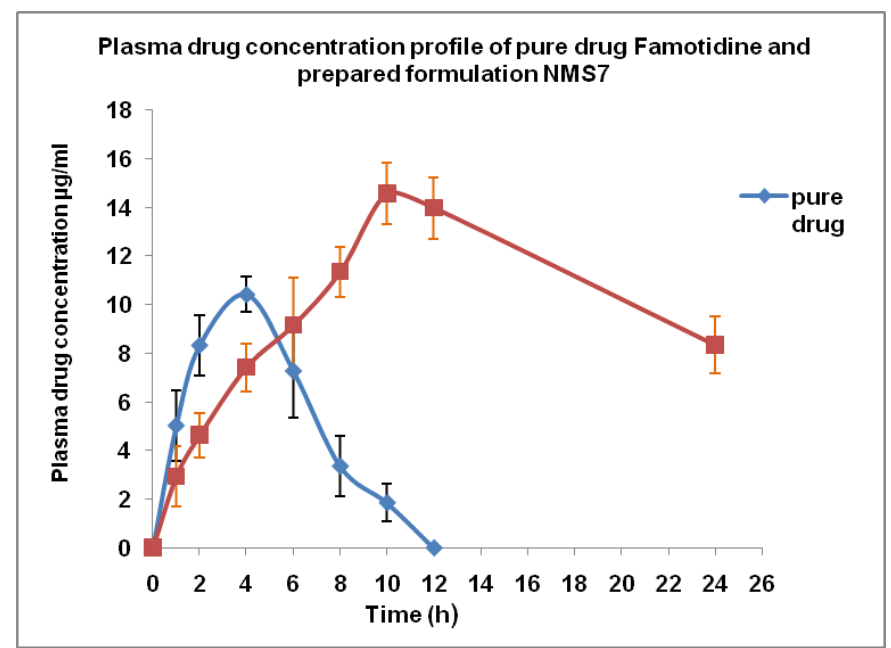

Fig. 4: Plasma drug concentration profile of pure drug famotidine and prepared niosomal formulation NMS7 of famotidine values represented as mean $\pm S D(n=3)$ and values are statistically significant $* P<0.05$

Table 5: Pharmacokinetic parameters of drug determined after administration of free drug solution and niosomal formulation

\begin{tabular}{llll}
\hline S. No. & Pharmacokinetic parameters & Free drug & NMS7 \\
\hline 1. & ${ }^{*} \mathrm{C}_{\max }$ & $10.427 \pm 0.74 \mu \mathrm{g} / \mathrm{ml}$ & $14.568 \pm 1.24 \mu \mathrm{g} / \mathrm{ml}$ \\
2. & $\mathrm{t}_{\max }$ & $4 \mathrm{~h}$ & $10 \mathrm{~h}$ \\
3. & AUC & $61.55 \mu \mathrm{g} / \mathrm{ml} \mathrm{h}$ & $242.765 \mu \mathrm{g} / \mathrm{ml} \mathrm{h}$ \\
\hline
\end{tabular}

*values are expressed as mean $n=3, \pm$ SD: standard deviation

\section{Stability studies}

\section{Drug leakage study}

It is clear from the results obtained that the niosomes have shown a minimum drug loss at refrigerated condition and fairly high retention of drug inside the vesicles was observed these results were concordant to Asthana et al. (2016) [5]. At this low temperature condition only $2.797 \%$ of drug was lost as compared to storage at
$25{ }^{\circ} \mathrm{C}$ and $37{ }^{\circ} \mathrm{C}$ which led to $4.113 \%$ and $5.125 \%$ drug loss respectively at the end of $35 \mathrm{~d}$ results shown in (table 6). The higher amount of drug leakage at elevated temperature may be related to the degradation of lipid constituting the bilayers resulting in defect in membrane packing and loss of overall rigidity that makes them leaky. So it can be concluded from the above discussion that the niosomal formulation should be stored at lower temperatures to minimize drug loss.

Table 6: Drug leakage studies of niosomal formulation NMS7 at various temperature conditions

\begin{tabular}{|c|c|c|c|c|c|c|}
\hline \multirow[t]{2}{*}{ Time(days) } & \multicolumn{2}{|l|}{$4^{\circ} \mathrm{C}$} & \multicolumn{2}{|l|}{$25^{\circ} \mathrm{C}$} & \multicolumn{2}{|l|}{$37^{\circ} \mathrm{C}$} \\
\hline & $\begin{array}{l}\text { \% Drug remaining } \\
\text { entrapped }\end{array}$ & $\begin{array}{l}\text { \% drug } \\
\text { lost }\end{array}$ & $\begin{array}{l}\text { \% Drug remaining } \\
\text { entrapped }\end{array}$ & $\begin{array}{l}\text { \% drug } \\
\text { lost }\end{array}$ & $\begin{array}{l}\text { \% Drug remaining } \\
\text { entrapped }\end{array}$ & $\begin{array}{l}\text { \% drug } \\
\text { lost }\end{array}$ \\
\hline Initialload & 100 & - & 100 & - & 100 & - \\
\hline 7 & 99.529 & 0.472 & 98.517 & 1.483 & 98.113 & 1.887 \\
\hline 14 & 98.7195 & 1.281 & 97.405 & 2.596 & 96.898 & 3.101 \\
\hline 21 & 98.214 & 1.786 & 97.000 & 3.000 & 96.292 & 3.708 \\
\hline 28 & 97.607 & 2.393 & 96.696 & 3.303 & 95.988 & 4.012 \\
\hline 35 & 97.203 & 2.797 & 95.887 & 4.113 & 94.876 & 5.125 \\
\hline
\end{tabular}




\section{CONCLUSION}

The present research was conducted to build up an appropriate drug delivery system for delivery of famotidine. The niosomal suspension of famotidine was successfully prepared from thin film hydration technique. The characterization of the drug loaded niosomal suspension by SEM and TEM revealed spherical shape with homogienety in all optimized formulations with polydispersity values well suited for oral drug delivery, zeta potential and vesicle size values reveal that the formulations have good stability with vesicle size in nano range. The kinetics of drug release reveal that the formulations show a better fit to zero order release pattern which predicts a controlled release pattern of drug from niosomes. The In vivo study clearly shows a higher Cmax value as compared to free drug which is indicative of improvement in bioavailability furthermore the AUC obtained in case of best formulation clearly shows a fourfold increase which shows a prolonged duration of drug release for more than $24 \mathrm{~h}$ making the formulation suitable for once a day administration thus improving the patient compliance and reducing the problems associated with peak and valley curves in plasma drug concentrations. The stability studies confirm that the formulations are most stable when kept at lower temperature. Thus it can be concluded that the niosomes can be successfully employed for prolonging the drug release and can prove to be a better drug delivery option.

\section{ACKNOWLEDGEMENT}

The authors are thankful to CDRI Lucknow for providing the TEM facility, central instrument facility at IIT-BHU(Banaras Hindu university) for SEM facility, authors are also thankful to Department of Pharmaceutics, Monad University Hapur.

\section{FUNDING}

Nil

\section{AUTHORS CONTRIBUTIONS}

The research work was conducted under the guidance of $\mathrm{Dr}$ Raghuveer Irchhaiya and the research work and manuscript preparation was done by Rizwana Khan.

\section{CONFLICT OF INTERESTS}

The authors of the present publication receive no funding for this research work, the authors Rizwana Khan and Raghuveer Irchhaiya have no conflict of interests.

\section{REFERENCES}

1. Lamprecht A. Nanotherapeutics: drug delivery concepts in nanoscience. $1^{\text {st }}$ ed. Singapore: Pan Stanford publishing; 2009.

2. Mokale VJ, Patil HI, Patil AP, Shirude PR, Naik JB. Formulation and optimisation of famotidine proniosomes: an in vitro and ex vivo study. J Exp Nanosci 2016;11:97-110.

3. Khan R, Irchhaiya R. Niosomes: a potential tool for novel drug delivery. J Pharm Investig 2016;46:195-204.

4. Prescott LF, Nimmo WS. Novel drug delivery and its therapeutic applications. New York: John Wiley and sons Inc; 1989.

5. Asthana GS, Sharma PK, Asthana A. In vitro and in vivo evaluation of niosomal formulation for controlled delivery of clarithromycin. Scientifica 2016. http://dx.doi.org/10.1155/ 2016/6492953

6. Vyas J, Vyas P, Sawant K. Formulation and evaluation of topical niosomal gel of erythromycin. Int J Pharm Pharm Sci 2011;3:123-6.

7. Ammar HO, Ghorab M, El-Nahhas SA, Higazy IM. Proniosomes as a carrier system for transdermal delivery of tenoxicam. Int J Pharm 2011;405:142-52.

8. Mokhtar M, Sammour OA, Hammad MA, Megrab NA. Effect of some formulation parameters on flurbiprofen encapsulation and release rates of niosomes prepared from proniosomes. Int J Pharm 2008;361:104-11.

9. Kumar BS, Krishna R, Lakshmi PS, Vasudev DT, Nair SC. Formulation and evaluation of niosomal suspension of cefixime. Asian J Pharm Clin Res 2017;10:194-201.
10. Balakrishnana P, Shanmugam S, Lee WS. Formulation and in vitro assessment of minoxidil niosomes for enhanced skin delivery. Int J Pharm 2009;377:1-8.

11. Mukherjee B, Patra B, Layek B, Mukherjee A. Sustained release of acyclovir from nano-liposome and nano-niosomes: an in vitro study. Int J Nanomed 2007;2:213-25.

12. Ruckmani K, Jayakar B, Ghosal SK. Nonionic surfactant vesicles(niosomes) of cytarabine hydrochloride for effective treatment of leukemias: encapsulation, storage, and in vitro release. Drug Dev Ind Pharm 2000;26:217-22.

13. Aggarwal D, Kaur IP. Improved pharmacodynamics of timolol maleate from a mucoadhesive niosomal ophthalmic drug delivery system. Int J Pharm 2005;290:155-9.

14. Omaima N El Gazayerly, Ahmed HH. Preparation and evaluation of acetazolamide liposomes as an ocular delivery system. Int J Pharm 1997;158:121-7.

15. Junyaprasert VB, Manwiwattanakul G. Release profile comparison and stability of diltiazem-resin microcapsules in sustained release suspensions. Int J Pharm 2008;352:81-91.

16. El-Samaligy MS, Afifi NN, Mahmoud EA. Increasing bioavailability of silymarin using a buccal liposomal delivery system: preparation and experimental design investigation. Int J Pharm 2006;308:140-8.

17. Ritger PL, Peppas NA. A simple equation for description of solute release I. Fickian and non-fickian release from nonswellable devices in the form of slabs, spheres, cylinders or discs. J Controlled Release 1987;5:23-36.

18. Pandey H, Parashar V, Parashar R, Prakash R, Ramteke PW, Pandey AC. Controlled drug release characteristics and enhanced antibacterial effect of graphene nanosheets containing gentamicin sulfate. Nanoscale 2011;11:29-36.

19. Kamboj S, Saini V, Bala S. Formulation and characterization of drug loaded nonionic surfactant vesicles (Niosomes) for oral bioavailability enhancement. Sci World J 2014:1-8. http://dx.doi.org/10.1155/2014/959741

20. Sharma UK, Verma A, Prajapati SK, Pandey H, Pandey AC. In vitro, in vivo and pharmacokinetic assessment of amikacin sulphate laden polymeric nanoparticles meant for controlled ocular drug delivery. Appl Nanosci 2015;5:143-55.

21. Jayakrishnan A, Joseph NJ, Lakshmi S. A floating type oral dosage form for peroxicam based on hollow polycarbonate microspheres: in vitro and in vivo evaluation in rabbits. J Controlled Release 2002;79:71-9.

22. Mastiholimath VS, Dandagi PM, Gadad AP, Mathews R, Kulkarni AR. In vitro in vivo evaluation of ranitidine hydrochloride ethyl cellulose floating microparticles. J Microencapsulation 2008;25:307-14.

23. Mishra V, Kaur R. Formulation and pharmacokinetic study of famotidine loaded microballons. Int J Pharm Pharm Sci 2012;4:511-5.

24. Azeem A, Anwer MK, Talegaonkar S. Niosomes in sustained and targeted drug delivery: some recent advances. J Drug Target 2009;17:671-89.

25. Hao YM, Li K. Entrapment and release difference resulting from hydrogen bonding interactions in niosomes. Int $\mathrm{J}$ Pharm 2011;403:245-53.

26. Yoshioka T, Sternberg B, Florence AT. Preparation and properties of vesicles (niosomes) of sorbitan monoesters (Span 20, 40, 60 and 80) and a sorbitan triester (Span 85). Int J Pharm 1994; $105: 1-6$

27. Kibbe AH. Handbook of pharmaceutical excepients. Washington DC; 2000.

28. Biswal S, Murthy PN, Sahu J, Sahoo P, Amir F. Vesicles of nonionic surfactants (niosomes) and drug delivery potential. Int J Pharm Sci Nanotechnol 2008;1:1-8.

29. Guinedi AS, Mortada ND, Mansour S, Hathout RM. Preparation and evaluation of reverse-phase evaporation and multilamellar niosomes as ophthalmic carriers of acetazolamide. Int J Pharm 2005;306:71-82.

30. Hao Y, Zhao F, Li N, Yang Y, Li K. Studies on high encapsulation of cholchicine by a noisome system. Int J Pharm 2002;224:73-80.

31. Bayindir ZS, Yuksel N. Characterization of niosomes prepared with various non ionic surfactants for paclitaxel oral delivery. J Pharm Sci 2010;99:2049-60. 
32. Nasr M, Mansour S, Mortada ND, Elshamy AA. Vesicular aceclofenac systems: a comparative study between liposomes and niosomes. J Microencapsul 2008;25:499-512.

33. Saraswathi TS, Mothilal M, Jaganathan MK. Niosomes as an emerging formulation tool for drug delivery-a review. Int J Appl Pharm 2019;11:7-15.

34. Badran M. Formulation and in vitro evaluation of flufenamic acid loaded deformable liposome for improved skin delivery. Digest J Nanomater Biostruct 2014;9:83-91.
35. Chen M, Liu X, Fahr A. Skin penetration and deposition of carboxyfluorescein and temoporfin from different lipid vesicular systems: in vitro study with finite and infinite dosage application. Int J Pharm 2011;408:223-34.

36. Putri DCA, Dwiastuti $R$, Marchaban $M$, Nugroho AK. Optimization of mixing temperature and sonication duration in liposome preparation.J Pharm Sci Commun 2017;14:79-85. 